Mini Review

\title{
Marine invasive alien crustaceans of India
}

Volume 5 Issue 2 - 2017

\section{Mini Review}

An invasive species is one which has been introduced from one geographical location to another through human agency and upon establishment has a tendency to spread (invade), causing damage to the environment, man-made structure and human health or economy. An introduced (also referred to as alien, exotic, non-indigenous or non-native) species becomes invasive when it outcompetes native species for various resources such as space, light, water or food. The new environment may offer fewer able competitors, thereby allowing the invader species to multiply quickly (Wikipedia). The invasives are widely distributed in all types of ecosystems and posing a serious threat to native biodiversity leading to the extinction of native species. Wilcove et al. ${ }^{1}$ considered invasive species as the second major cause of extinctions of native and endemic species around the world. According to an estimate in 2001, the economic loss caused by invasive species to agriculture and forestry in India is to the tune of 91 billion dollar a year Hiremath \& Krishnan. ${ }^{2}$ The magnitude of invasions may be apparently local, but the drivers of bio-invasion are global. According to the Convention on Biological Diversity (CBD), there is a need for "compilation and dissemination of information on alien species that threaten ecosystem, habitats, or species, to be used in the context of any prevention, introduction and mitigation activities (CBD, 2000)".

Invasion of a species (bio-invasion) from one bio-region to another takes place in two ways. They may be intentional (such as aquacultural/agricultural purposes etc.) or unintentional (such as through ship's ballast water discharge and fouling of ship hulls).

India has 13 major and 200 non-major ports. About 95\% of India's trade by volume and $70 \%$ of value takes place through maritime transport. Ships are considered as the major factor for translocation of alien species from one bio-region to another. In this respect, harbours

\section{NC Nandi, MK Dev Roy}

Social Environmental and Biological Association, India

Correspondence: NC Nandi, Social Environmental and Biological Association, India,

Email nepalchandra.nandi@gmail.com

Received: February 02, 2017 | Published: February 13, 2017 serve as a gateway for the introduction of species. Information on marine invasive species from Indian maritime system is very meagre. During the last one decade, quite a good number of crustacean species has been reported as 'introduced' to Indian waters by ballast water (Table 1). This consists of 5 species of decapods, 6 species of isopods, 9 species of amphipods, 7 species of cirripedes and 5 species of copepods. Only one species of decapod, namely, Litopenaeus vannamei has been introduced to Indian water for aquaculture purposes. The invasive status of some of these species, however, are yet to be determined. So far, no ecological impact of these species to native ecosystems are ascertained. However, the possibility that they could be undergoing a lag phase cannot be ruled out. This is the first comprehensive list on crustacean invasive species from Indian maritime ecosystems..$^{3-11}$

Table I List of alien crustaceans recorded from Indian water and their threat stop

\begin{tabular}{|c|c|c|c|c|c|}
\hline \multicolumn{2}{|c|}{ SI. No.Group and Species } & \multirow[t]{2}{*}{ Family } & \multirow[t]{2}{*}{ Native/Exotic/Cryptogenic } & \multirow[t]{2}{*}{ Threat Status } & \multirow[t]{2}{*}{ Source } \\
\hline & Decapoda & & & & \\
\hline I & Penaeus (Penaeus) monodon (Fabricius, I 798) & Penaeidae & Native & Not suspected & Anil et al. ${ }^{4}$ \\
\hline 2 & Litopenaeus vannamei (Boone, I931) & Penaeidae & Exotic, Introduced & Not suspected & Dev Roy. ${ }^{5}$ \\
\hline 3 & Charybdis (Charybdis) feriata (Linnaeus, I758) & Portunidae & Native & Suspected harmful species & Anil et al. ${ }^{4}$ \\
\hline 4 & $\begin{array}{l}\text { Charybdis (Charybdis) hellerii (A.Milne } \\
\text { Edwards, I86I) }\end{array}$ & Portunidae & Native & Known harmful species & Anil et al. ${ }^{4}$ \\
\hline \multirow[t]{2}{*}{5} & Scylla serrata (Forskal, I775) & Portunidae & Native & Not suspected & Anil et al. ${ }^{4}$ \\
\hline & Isopoda & & & & \\
\hline 6 & Cirolana hardfordi (Lockington, I877) & Cirolanidae & Exotic & Not suspected & Anil et al. ${ }^{4}$ \\
\hline 7 & Cilicaea latreillei Leach Limnoriidae, I8I8 & Cirolanidae & Exotic & Not suspected & Anil et al. ${ }^{3}$ \\
\hline 8 & Paradella dianae (Menzies, I962) & Sphaeromatidae & Exotic & Not suspected & Anil et al. ${ }^{4}$ \\
\hline 9 & Sphaeroma serratum (Fabricius, I787) & Sphaeromatie & Exotic & Not suspected & Anil et al. ${ }^{4}$ \\
\hline 10 & Sphaeroma walkeri & Sphaeromatie & Native & Not suspected & Anil et al. ${ }^{4}$ \\
\hline \multirow[t]{2}{*}{11} & Synidotea laevidorsalis (Benedict, I897 & Idoteidae & Exotic & Not suspected & Anil et al. ${ }^{4}$ \\
\hline & Amphipoda & & & & \\
\hline 12 & $\begin{array}{l}\text { Monocorophium acherusicum (Costa, I853) } \\
\text { as Corophium acherusicum Costa, I } 853\end{array}$ & Corophiidae & Exotic & Not yet assessed & Shyamasudari.$^{8}$ \\
\hline
\end{tabular}


Table Continued..

\begin{tabular}{|c|c|c|c|c|c|}
\hline \multicolumn{2}{|c|}{ SI. No.Group and Species } & \multirow{2}{*}{$\begin{array}{l}\text { Family } \\
\text { Ischyroceride }\end{array}$} & \multirow{2}{*}{$\begin{array}{l}\text { Native/Exotic/Cryptogenic } \\
\text { Exotic }\end{array}$} & \multirow{2}{*}{$\begin{array}{l}\text { Threat Status } \\
\text { Not yet assessed }\end{array}$} & \multirow{2}{*}{$\begin{array}{l}\text { Source } \\
\text { Shyamasundari. }^{8}\end{array}$} \\
\hline 13 & Jassa falcata (Montague, I808) & & & & \\
\hline 14 & Jassa marmorata Holmes, 1905 & Ischyroceride & Exotic & Not suspected & Anil et al. ${ }^{4}$ \\
\hline 15 & Elasmopus rapax Costa, I853 & Maeridae & Exotic & Not yet assessed & Shyamasundari. ${ }^{8}$ \\
\hline 16 & $\begin{array}{l}\text { Quadrimaera pacifica (Schellenberg, 1938) as } \\
\text { Maera pacifica Schellenberg, } 1938\end{array}$ & Maeridae & Exotic & - & Anil et al. ${ }^{3}$ \\
\hline 17 & Paracaprella pusilla Mayr, I890 & Caprellidae & Exotic & Not yet assessed & Guerra-García, ${ }^{7}$ \\
\hline 18 & Stenothoe gallensis Walker, 1904 & Stenothoidae & Exotic & - & Anil et al. ${ }^{4}$ \\
\hline 19 & Stenothoe valida Dana, I852 & Stenothoidae & Exotic & Not yet assessed & Shyamasundari. ${ }^{8}$ \\
\hline \multirow[t]{2}{*}{20} & Podocerus brasiliensis (Dana, I853) & Podoceridae & Exotic & - & $\begin{array}{l}\text { Anil et al. }{ }^{4} \\
\text { Shyamasundari. }{ }^{8}\end{array}$ \\
\hline & Cirripedia & & & & \\
\hline 21 & $\begin{array}{l}\text { Amphibalanus cirratus (Darwin, 1854) } \\
\text { as Balanus amphitrite cirratus }\end{array}$ & Archaebalane & Native & Not suspected & Anil et al. ${ }^{4}$ \\
\hline 22 & $\begin{array}{l}\text { Amphibalanus eburneus (Gould, I84I) } \\
\text { as Balanus amphitrite eburneus }\end{array}$ & Archaebalane & Cryptogenic & Not suspected & Anil et al. ${ }^{4}$ \\
\hline 23 & $\begin{array}{l}\text { Amphibalanus reticulatus (Utinomi, 1967) } \\
\text { as Balanus reticulatus Utinomi, } 1967 \\
\text { and Balanus amphitrite hawaiiensis Broch }\end{array}$ & Archaebalane & Exotic & Not suspected & $\begin{array}{l}\text { Anil et al. }{ }^{4} \text { Anil } \\
\text { et al. }{ }^{3}\end{array}$ \\
\hline 24 & $\begin{array}{l}\text { Fistulobalanus pallidus (Darwin, } \\
\text { I854)= Balanus amphitrite stutsburi (Darwin) }\end{array}$ & Balanidae & Exotic & - & Wagh. ${ }^{9}$ Anil et al. ${ }^{4}$ \\
\hline 25 & Balanus trigonus Darwin, I 854 & Balanidae & Native & Not suspected & Anil et al. ${ }^{4}$ \\
\hline 26 & Megabalanus tintinnabulum (Linnaeus, I758) & Balanidae & Exotic & Known harmful species & Anil et al. ${ }^{4}$ \\
\hline \multirow[t]{2}{*}{27} & Megabalanus zebra (Darwin, I854) & Balanidae & Cryptogenic & Not suspected & Anil et al. ${ }^{4}$ \\
\hline & Copepoda & & & & \\
\hline 28 & Nannocalanus minor (Claus, I863) & Calanidae & Exotic & - & Gaonkar et al. ${ }^{6}$ \\
\hline 29 & Cosmocalanus sp. & Calanidae & - & - & Gaonkar et al. ${ }^{6}$ \\
\hline 30 & Paracalanus sp. & Calanidae & - & - & Gaonkar et al. ${ }^{6}$ \\
\hline 31 & Tortanus sp. & Tortanidae & - & - & Gaonkar et al. ${ }^{6}$ \\
\hline 32 & Euterpina acutifrons (Dana, I847) & Euterpinidae & Exotic & - & Gaonkar et al. ${ }^{6}$ \\
\hline
\end{tabular}

\section{Acknowledgments}

None.

\section{Conflicts of interest}

None.

\section{References}

1. Wilcove DS, Rothstein D, Dubow J, et al. Quantifying threats to imperiled species in the United States. Bioscience. 1998;48(8):607-615.

2. Hiremath AJ Krishnan S India knows its invasive species problem but this is why nobody can deal with it properly. The Wire: Environment. Convention on Biological Diversity. 2016

3. Anil AC, Venkat K, Sawant SS, et al. Marine bioinvasion: concern for ecology and shipping. Curr Sci. 2002;83(3):214-218.

4. Anil AC, Clarke C, Hayes T, et al. Ballast water risk assessment: Ports of Mumbai and Jawaharlal Nehru,India, October 2003: Final Report. IMO GloBallast Monograph Series. 2003;11:1-63. IMO, London.
5. Dev Roy MK Problems and prospects of White leg shrimp culture in India. SEBA Newsletter. 2007;4(1):15.

6. Gaonkar CA, Sawant SS, Anil AC, et al.Mumbai harbour, India: gateway for introduction of marine organisms. Environ Monit Assess. 2010;1631:583-589.

7. Guerra-García JM, Ganesh T, Jaikumar M, et al. Caprellids (Crustacea Amphipoda) from India. Helgoland Marine Research. 2010;64(4):297310

8. Shyamasundari K Amphipoda. In: Nagabhushanam R \& Thompson MF (Eds.), Fouling Organisms of the Indian Ocean. Biology and Control Technology pp. 1997;363-390.

9. Wagh $\mathrm{AB}$ Probable transportation of Balanus amphitrite stutsburi (Darwin) by ship. J Bombay nat Hist Soc. 1973;70(2):399-400.

10. Introduced species en.wikipedia.org/wiki/Introduced species

11. Invasive species en.wikipedia.org/wiki/Invasive species 